\title{
Ryu-Takayanagi formula for symmetric random tensor networks
}

\author{
Goffredo Chirco, Daniele Oriti, and Mingyi Zhang \\ Max Planck Institute for Gravitational Physics, Albert Einstein Institute, \\ Am Mühlenberg 1, 14476 Potsdam, Germany.
}

(Received 7 March 2018; published 8 June 2018)

\begin{abstract}
We consider the special case of random tensor networks (RTNs) endowed with gauge symmetry constraints on each tensor. We compute the Rényi entropy for such states and recover the Ryu-Takayanagi (RT) formula in the large-bond regime. The result provides first of all an interesting new extension of the existing derivations of the RT formula for RTNs. Moreover, this extension of the RTN formalism brings it in direct relation with (tensorial) group field theories (and spin networks), and thus provides new tools for realizing the tensor network/geometry duality in the context of background-independent quantum gravity, and for importing quantum gravity tools into tensor network research.
\end{abstract}

DOI: 10.1103/PhysRevD.97.126002

\section{INTRODUCTION}

Tensor networks [1] have developed into a powerful and ubiquitous formalism in quantum information and in the analysis of quantum many-body systems. It is first of all a very efficient way to capture the entanglement properties of such many-body systems, as well as quantum field theories (including lattice gauge theories), and it provides a general framework for describing (and identifying) quantum states characterized by area laws, which indeed include the ground states of several interesting quantum many-body systems [2]. Somewhat surprisingly, it also provides a natural framework for investigating the nature of spacetime at the Planck scale. This comes about from two main directions. First, different theoretical frameworks, from background-independent quantum gravity to string theory, hint at a scenario, where continuum spacetime geometry is replaced, at a more fundamental level, by pregeometric quantum degrees of freedom, often of purely combinatorial and algebraic nature. In (tensorial) group field theories (GFTs) [3] and random tensor models [4], as well as in loop quantum gravity (LQG) and spin foam models [5], pregeometric quantum degrees of freedom are encoded in random combinatorial network structures, labeled by algebraic data. In particular, they are encoded in spin networks, graphs labeled by irreps of $S U(2)$ and endowed with a gauge symmetry at each node. These type of quantum states, in fact, are very close to tensor networks [6], and tensor network techniques have found already a number of quantum gravity applications [7]. A

Published by the American Physical Society under the terms of the Creative Commons Attribution 4.0 International license. Further distribution of this work must maintain attribution to the author(s) and the published article's title, journal citation, and DOI. Funded by SCOAP . discrete spacetime and geometry is naturally associated, at a semiclassical level, to such structures and their quantum dynamics is directly related to (noncommutative) discrete gravity path integrals [8]. The outstanding issue is then to show the emergence of continuum spacetime and geometry from the full quantum dynamics of the same pregeometric degrees of freedom, which in fact describe a quantum spacetime as a peculiar sort of quantum many-body system [9]. It is natural to expect that the entanglement between the fundamental entities constituting spacetime is crucial for its emergence and for understanding the quantum nature of geometry at the Planck scale, and thus that tensor network techniques will provide relevant tools in this context. Second, a different, but probably related relation between entanglement and geometry has been unraveled in the context of holographic gauge/gravity duality, and also here tensor network states recently acquired a prominent role. One key example is the Ryu-Takayanagi (RT) formula, which relates the entanglement entropy of a region of the boundary gauge field to the area of the minimal surface in the bulk homologous to the same region [10]. The same holographic area scaling behavior has been reproduced in the so-called anti-de Sitter/multiscale entanglement renormalization ansatz (AdS/MERA) scheme [11], where the hyperbolic structure of the MERA tensor network algorithm used to represent the vacuum of a boundary lattice conformal field theory state is directly associated to the dual spatial geometry.

More recently, a lot of interest focused on random tensor network (RTN) states, a class of algorithms shown to satisfy the Ryu-Takayanagi formula, as well as quantum error correction properties, in the large-bond-dimension limit [12]. Networks of random pure tensor states are nearly maximally entangled states [13] and can be used as toy models of a thermal state $[14,15]$. This allows to use statistical field theory techniques to compute the typical entropies and other 
quantities of interest for these states via the evaluation of partition functions of classical statistical models [12].

In this particular context, the generalization of the tensor network decomposition techniques in terms of GFTs provided in Ref. [6], allows to map specific GFT partitions functions to tensor network states with a specific probability measure, providing a direct relation between the auxiliary tensor network bulk geometry and discrete gravity.

Along this direction, the present work aims at extending the formalism of RTN to incorporate one key feature of the random networks appearing in quantum gravity-the gauge symmetry constraint [16] — and deriving the RT formula in this extension.

The paper is organized as follows. In the next section, we recall the definition of tensor network states, and that of their random version. Then, we introduce the symmetric random tensor networks that we use in the rest of the paper. Having done so, we compute the second Rényi entropy for random tensor networks endowed with a local gauge symmetry constraint and derive the RT formula for them. We end with some concluding remarks.

\section{TENSOR NETWORK STATES AND HOLOGRAPHIC BEHAVIOR}

A tensor network is a collection of tensors associated to nodes of a network, connected by contraction operations, associated to links of the same network.

Generically, a rank- $v$ (or $v$-valent) tensor $T$ is a multidimensional array of complex numbers with $v$ indices $\lambda$, each taking values from a set of dimension ("size") $D_{|\lambda|} \in$ $\mathbb{N}^{+}$[16]. For simplicity, all indices are assumed to have the same size $D_{|\lambda|}=D$.

At the quantum level, to each leg of the tensor one associates a Hilbert space $\mathbb{U}_{D}$, with dimension $D$, so that a covariant tensor of rank $v$ is a multilinear form on the Hilbert space of the vertex $T: \mathbb{U}_{n} \equiv \mathbb{T}_{D}^{\otimes v}$. Given an orthonormal basis $\left|\lambda_{n}\right\rangle, n=1, \ldots, D$ in $\mathbb{H}_{D}$, we can generally define a tensor state as

$$
|T\rangle=\sum_{\lambda_{1}, \ldots \lambda_{d}} \hat{T}_{\lambda_{1} \ldots \lambda_{v}}\left|\lambda_{1}\right\rangle \otimes \cdots \otimes\left|\lambda_{v}\right\rangle,
$$

and identify the tensor with the state components

$$
\hat{T}_{\lambda_{1} \ldots \lambda_{v}} \equiv T\left(\lambda_{1}, \ldots, \lambda_{v}\right) \text {. }
$$

We can then represent any such tensor state as a single vertex graph with $v$ open links emanating from it.

A state corresponding to a set of unconnected vertices is given by a tensor product of individual tensor states

$$
\left|\mathcal{T}_{\mathcal{N}}\right\rangle \equiv \underset{n}{\otimes}\left|T_{n}\right\rangle
$$

In a connected network graph, individual tensor states are glued by links, to each end of which we associate a Hilbert space $\mathbb{U}_{D}$. The Hilbert space of the link $\ell$ is then $\mathbb{H}_{\ell}=\mathbb{T}_{D}^{\otimes 2}$ while a link state can be generally written as

$$
|M\rangle=M_{\lambda_{1} \lambda_{2}}\left|\lambda_{1}\right\rangle \otimes\left|\lambda_{2}\right\rangle
$$

where the coefficients $M_{\lambda_{1} \lambda_{2}}$ indicate generic quantum correlations between the links ends. ${ }^{1}$ One could picture this gluing as the joining of two of the open links emanating from the original vertices (along their open ends), to form a link of the resulting network.

The entanglement of links encode the information on the connectivity of the graph: two nodes are connected if their corresponding states contract with an entangled link state,

$$
\begin{aligned}
\left|\mathcal{T}_{12}\right\rangle & \equiv\left\langle M|| T_{1}\right\rangle\left|T_{2}\right\rangle \\
& =T_{\lambda_{1} \ldots \lambda_{a} \ldots \lambda_{v}}^{(1)} \bar{M}_{\lambda_{a} \lambda_{b}} T_{\lambda_{1}^{\prime} \ldots \lambda_{b} \ldots \lambda_{u}^{\prime}}^{(2)} \underset{i \neq a}{\otimes}\left|\lambda_{i}\right\rangle \otimes \underset{j \neq b}{\bigotimes}\left|\lambda_{i}^{\prime}\right\rangle .
\end{aligned}
$$

Accordingly, given a network $\mathcal{N}$ with $N$ nodes and $L$ links, the corresponding tensor network state is given by the contraction

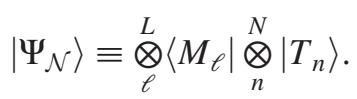

As all but the open links of the network are contracted with nodes, $\left|\Psi_{\mathcal{N}}\right\rangle$ can be thought of as an element of the Hilbert space $\mathbb{U}_{\partial \mathcal{N}}$ associated to the open links comprising the boundary of the network graph.

In lattice gauge theory, formulated in terms of tensor networks, the open links carry the physical degrees of freedom of the theory, while the fully contracted internal graph is interpreted as a virtual structure, whose correlation structure can be tuned to match the desired properties of the boundary lattice state $\left|\Psi_{\mathcal{N}}\right\rangle$ in $\mathbb{\boxplus}_{\partial \mathcal{N}}$ [17]. In quantum gravity formalisms based on spin networks (and thus on tensor networks), like GFT and LQG, the internal graphs carry the degrees of freedom of a spatial manifold, while the open links are associated to its boundary (corners of the

\footnotetext{
${ }^{1}$ One can observe it by defining a density matrix $\rho_{M} \equiv|M\rangle\langle M|$ and tracing out one of the Hilbert spaces (without losing generality, tracing out $\mathbb{W}_{D}$ of $\left|\lambda_{2}\right\rangle$ ), and then computing the von Neumann entropy of the reduced density matrix $\rho_{1} \equiv \operatorname{Tr}_{2} \rho_{M}=M^{\dagger} M$. The entropy $S=\operatorname{Tr} \rho_{1} \ln \rho_{1}$ is nonzero unless $M_{\lambda_{1} \lambda_{2}}$ can be split as $M_{\lambda_{1} \lambda_{2}}=A_{\lambda_{1}} B_{\lambda_{2}}$. Notice that the definition in Eq. (4) provides a slightly more general version of a link state than that used in the tensor network literature. There a link usually does not carry additional structure which means that indices in connected tensors get identified and summed over. For simplicity, in the next sections we will also assume that the link state is maximally entangled, i.e.,

$$
|M\rangle=\frac{1}{\sqrt{D}} \delta_{\lambda_{1} \lambda_{2}}\left|\lambda_{1}\right\rangle \otimes\left|\lambda_{2}\right\rangle
$$

The latter corresponds to the simplest type of gluing-imposing gauge invariance-in the spin network states used in quantum gravity, and forming indeed a special type of tensor networks $[6,16]$.
} 
spacetime manifold) and carry indeed additional degrees of freedom, related to the breaking of diffeomorphism symmetry on the same boundary [18].

\section{A. Random tensor network states}

Recently, a lot of interest has focused on the study of networks of large-dimensional random tensors (RTNs).

A convenient way to deal with RTNs is to consider the tensors $\hat{T}_{\lambda_{1} \ldots \lambda_{v}}$ on each node as unit complex vectors $T_{\mu}$ chosen independently at random in their respective Hilbert spaces $\mathbb{U}_{T} \simeq \mathbb{U}_{D}^{\otimes v}$ ( $D^{v}$-dimensional vector spaces), with inner product $\bar{T}_{\mu} T_{\mu}^{\prime}$. One can represent these vectors by choosing a map such that $T_{\mu} \equiv T_{\lambda_{1} \ldots \lambda_{v}}$, with

$$
\mu=\sum_{a=1}^{v} \lambda_{a} D^{v-a}=0,1, \ldots, D^{v}-1
$$

Moreover, with $|T\rangle \in \mathbb{U}_{n}$ being normalized, one has as well

$$
\langle T \mid T\rangle=\bar{T}_{\lambda_{1} \ldots \lambda_{v}} T_{\lambda_{1} \ldots \lambda_{v}}=\bar{T}_{\mu} T_{\mu}=1 .
$$

Notice also that the Hilbert space $\mathbb{U}_{T}$ corresponds to the fundamental representation of the group $\mathrm{U}\left(D^{v}\right)$. Given an arbitrary reference state $T_{\mu}^{0}$, a group element $U \in \mathrm{U}\left(D^{v}\right)$ will transform $T_{\mu}^{0}$ to a new vector $T_{\mu}^{U} \equiv\left(U T^{0}\right)_{\mu}$. A random tensor $T_{\lambda_{1} \ldots \lambda_{v}}$ corresponds then to a random choice of the group element $U \in \mathrm{U}\left(D^{v}\right)$ defining $T_{\mu}^{U}$, where the group element $U_{n}$ is independently chosen for each node of the network.

Idealized versions of RTNs, so-called pluperfect tensors, have been used to define bidirectional holographic codes, which simultaneously satisfy the RT formula for a subset of boundary states, error correction properties of bulk local operators, a kind of bulk gauge invariance, and the possibility of sub-AdS locality.

More recently, in particular, building on the statistical properties of large-dimensional random tensor networks, the technique of random state averaging was used in Ref. [12] to compute Rényi entropies and other quantities of interest in the corresponding tensor network states, by means of a mapping to the evaluation of partition functions of classical statistical models, like generalized Ising models with boundary pinning fields.

In what follows, we shall reconsider the random state averaging technique for a specific class of largedimensional random tensor networks endowed with extra symmetry constraints.

\footnotetext{
${ }^{2}$ The random average of an arbitrary function $f(|V x\rangle)$ of the state $\left|V_{x}\right\rangle$ is equivalent to an integration over $U$ according to the Haar probability measure $\int \mathrm{d} U f\left(U\left|0_{x}\right\rangle\right)$, with normalization $\int \mathrm{d} U=1$.
}

\section{RANDOM TENSOR NETWORK STATES WITH GAUGE SYMMETRY CONSTRAINTS}

Now let us consider a tensor $T_{\lambda_{1} \ldots \lambda_{v}}$ which satisfies the following symmetry:

$$
T_{\lambda_{1} \ldots \lambda_{v}}=T_{\left[\lambda_{1}+\ell\right]_{D} \ldots\left[\lambda_{v}+\ell\right]_{D}}, \quad \forall \ell \in \mathbb{Z} .
$$

The square bracket $[\cdots]_{D}$ denotes the modular arithmetic: for all $k \in \mathbb{Z}$ and $D \in \mathbb{Z}^{+}$

$$
[k]_{D} \equiv k \bmod D, \quad[k]_{D} \in \mathbb{Z} / D
$$

where $\mathbb{Z} / D$ is the set of integers modulo $D$. The symmetry in Eq. (10) is described by the $Z_{D}$ group. The Fourier transform of $T$ is given by the intertwiner of the $Z_{D}$ group. Such a tensor can be considered as a particular case of a GFT tensor field, with arguments/indices taking values on a finite group.

For all $k_{1}, k_{2} \in \mathbb{Z}$ and $D \in \mathbb{Z}^{+}$, it satisfies

$$
\begin{aligned}
{\left[k_{1}\right]_{D}+\left[k_{2}\right]_{D} } & =\left[k_{1}+k_{2}\right]_{D}, \\
{\left[\left[k_{1}\right]_{D}+\left[k_{2}\right]_{D}\right]_{D} } & =\left[k_{1}+k_{2}\right]_{D}, \\
{[D]_{D} } & =0 .
\end{aligned}
$$

In the presence of the symmetry (10), only $D^{v-1}$ components are independent. We choose a new map

$$
\mu=\lambda_{1} D^{v-1}+\sum_{a=2}^{v}\left[\lambda_{a}-\lambda_{1}\right]_{D} D^{v-a}
$$

such that

$$
T_{\mu}=\left(\begin{array}{c}
T_{\mu_{0}} \\
T_{\mu_{1}} \\
\vdots \\
T_{\mu_{D-1}}
\end{array}\right) \equiv T_{\lambda_{1} \ldots \lambda_{v}}
$$

where $T_{\mu_{i}}$ are $D^{v-1}$-dimensional vectors and

$$
T_{\mu_{i}}=T_{\mu_{j}}, \quad \forall i, j=0,1, \ldots, D-1 .
$$

From now on, for simplicity, we denote the tensor with the symmetry (10) with two indices: $\mu$ and $i=\lambda_{1} \in \mathbb{Z} / D$ as $T_{\mu_{i}}=T_{\lambda_{1} \ldots \lambda_{v}}$, with

$$
\mu_{i}=\sum_{a=2}^{v}\left[\lambda_{a}-\lambda_{1}\right]_{D} D^{v-a} \in \mathbb{Z} / D^{v-1} .
$$

For a given $i$, the vector $T_{\mu_{i}}$ is lying on a $D^{v-1}$ dimensional space, which is a fundamental representation space of the group $\mathrm{U}\left(D^{v-1}\right)$. Because $\bar{T}_{\mu} T_{\mu}=1$ and given Eq. (10), $T_{\mu_{i}}$ is also normalized

$$
\bar{T}_{\mu_{i}} T_{\mu_{i}}=D^{-1}, \quad \forall i=0,1, \ldots, D-1 .
$$


Then the random nature of the tensor $T_{\lambda_{1} \ldots \lambda_{v}}$ implies that, with respect to the same $T_{0}^{\mu}$, the group element $U_{n} \in$ $\mathrm{U}\left(D^{v-1}\right)$ is randomly chosen for each node.

\section{ENTANGLEMENT ENTROPY OF A RTN SUBREGION}

For the specific class of RTN states defined in Sec. III, we shall investigate the effect of the symmetry constraint on the holographic behavior of the entanglement entropy. We follow a similar procedure as in Ref. [12].

Given our tensor network state,

$$
\left|\Psi_{\mathcal{N}}\right\rangle \equiv \underset{\ell}{\otimes}\left\langle M_{\ell}\left|\stackrel{\otimes}{n}_{n}^{N}\right| T_{n}\right\rangle \in \mathbb{H}_{\partial \mathcal{N}}
$$

with tensor states satisfying the relation given in Eq. (10), we consider a bipartition of the boundary Hilbert space

$$
\mathbb{H}_{\partial \mathcal{N}}=\mathbb{H}_{A} \otimes \mathbb{H}_{B}
$$

associated to the definition of two (a priori not adjacent) boundary subregions $A$ and $B$ (see Fig. 1).

A measure of the entanglement between the two subsystems is given by the von Neumann entropy of the reduced density matrix of the subsystem, either $A$ or $B$, defined by partial tracing over the full system Hilbert space. Focusing on subsystem $A$, for $\rho \equiv\left|\Psi_{\mathcal{N}}\right\rangle\left\langle\Psi_{\mathcal{N}}\right|$, we have

$$
\rho_{A}=\operatorname{Tr}_{B}(\rho)
$$

and the entanglement entropy between $A$ and $B$ is given by the von Neumann entropy

$$
S_{\mathrm{EE}}(A)=-\operatorname{Tr}\left[\hat{\rho}_{A} \ln \hat{\rho}_{A}\right],
$$

where now

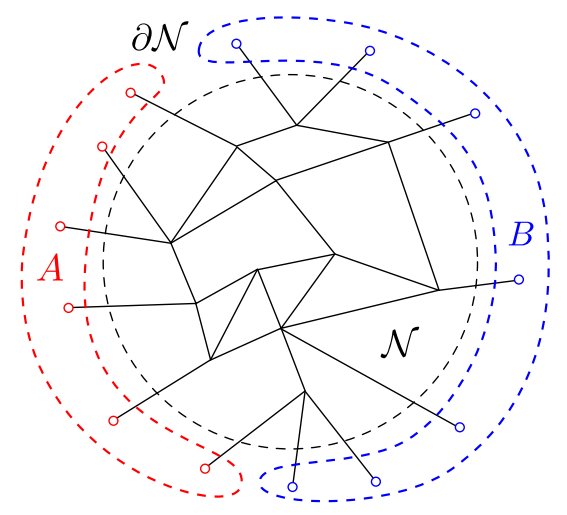

FIG. 1. Boundary $\partial \mathcal{N}$ of network $\mathcal{N}$ divided into two parts $A$ and $B$.

$$
\hat{\rho}_{A} \equiv \frac{\rho_{A}}{\operatorname{Tr} \rho}
$$

is the normalized reduced density matrix.

Following Ref. [12], we compute the entanglement between $A$ and $B$ in terms of the Rényi entropy. Contracting $N$ copies of the reduced density matrix $\rho_{A}$ and taking the logarithm of the trace of $\rho_{A}^{N}$, one obtains the $N$ th-order Rényi entropy

$$
S_{N}(A)=-\frac{1}{N-1} \ln \operatorname{Tr} \hat{\rho}_{A}^{N} \equiv-\frac{1}{N-1} \ln \frac{\operatorname{Tr} \rho_{A}^{N}}{(\operatorname{Tr} \rho)^{N}} .
$$

In the limit $N \rightarrow 1$, the Rényi entropy $S_{N}$ coincides with the von Neumann entropy of region $A$,

$$
S_{\mathrm{EE}}(A)=\lim _{N \rightarrow 1} S_{N}(A)
$$

and thus it provides a convenient measure of the entanglement entropy between regions $A$ and $B$.

We define the partition functions for the $N$ replicas of the reduced and full boundary states by

$$
\begin{aligned}
& Z_{A}^{(N)} \equiv \operatorname{Tr} \rho_{A}^{N}=\operatorname{Tr}\left[\rho^{\otimes N} \mathbb{P}\left(\pi_{A}^{0} ; N, d\right)\right], \\
& Z_{0}^{(N)} \equiv(\operatorname{Tr} \rho)^{N},
\end{aligned}
$$

where $\mathbb{P}\left(\pi_{A}^{0} ; N, d\right)$ is the 1 -cycle permutation operator acting on the states in region $A$,

$$
\mathbb{P}\left(\pi_{A}^{0} ; N, d\right)=\prod_{s=1}^{N} \delta_{\mu_{A}^{\left([s+1]_{N}\right)} \mu_{A}^{(s)},}
$$

$d$ is the dimension of the Hilbert space in the same region $A$, and $\mu^{(s)}$ is the sth replica of the index. In general, $\mathbb{P}(\pi ; N, d)$ denotes the $d$-dimensional representation matrix of permutation element $\pi$ in the $N$-permutation group $\mathcal{S}_{N}$.

With respect to the original open graph, described by the state $\rho \equiv\left|\Psi_{\mathcal{N}}\right\rangle\left\langle\Psi_{\mathcal{N}}\right|$, the two partition functions $Z_{A}^{(N)}, Z_{0}^{(N)}$ define new tensor network observables associated to closed graphs obtained by a specific trace scheme.

\section{A. Derivation of the second Rényi entropy}

As a first step, let us write down the second-order Rényi entropy, $S_{2}$, for a generic tensor network state $\left|\Psi_{\mathcal{N}}\right\rangle$ in $\mathbb{U}_{\partial \mathcal{N}}$. We rewrite states in the following synthetic index notation:

$$
\begin{aligned}
& \left|\Psi_{\mathcal{N}}\right\rangle \Leftrightarrow \Psi_{\left\{\lambda_{A}\right\}\left\{\lambda_{B}\right\}} \equiv \Psi_{A B}, \\
& \underset{n}{\otimes}\left|T_{n}\right\rangle \Leftrightarrow\left(\otimes_{n} T_{n}\right)_{\left\{\lambda_{A}\right\}\left\{\lambda_{B}\right\}\left\{\lambda_{C}\right\}} \equiv \mathcal{T}_{A B C}, \\
& \underset{\ell}{\otimes}\left\langle M_{\ell}\right| \Leftrightarrow\left(\underset{\ell}{\otimes} \bar{M}_{\ell}\right)_{\left\{\lambda_{C}\right\}} \equiv \overline{\mathcal{M}}_{C} .
\end{aligned}
$$


Based on the definition (7), the tensor network state is rewritten as

$$
\Psi_{A B}=\overline{\mathcal{M}}_{C} \mathcal{T}_{A B C}
$$

where $A$ and $B$ label the two regions of the boundary $\partial \mathcal{N}$.

All internal links are contracted with nodes. The density matrix corresponding to $\Psi_{A B}$ is

$$
\rho_{\bar{A} A \bar{B} B}=\bar{\Psi}_{\overline{A B}} \Psi_{A B} .
$$

Then $S_{2}$ is defined as

$$
\mathrm{e}^{-S_{2}}=\frac{Z_{A}^{(2)}}{Z_{0}^{(2)}}
$$

with $Z_{0}^{(2)}=\operatorname{Tr}\left[\rho^{\otimes 2}\right]$ and $Z_{A}^{(2)}=\operatorname{Tr}\left[\rho_{A}^{\otimes 2}\right]$.

In particular, for $N=2$, the cyclic group $\mathcal{S}_{n}$ only has two elements - the identity 1 and swap operator $\mathbb{F}$ - so that

$$
\mathbb{P}\left(\pi_{A}^{0} ; 2, d\right) \equiv \mathbb{F}^{(A)} \equiv \prod_{s=1}^{2} \delta_{\mu_{A}^{\left.(s+1]_{2}\right)} \mu_{A}^{(s)}}
$$

Then

$$
\begin{aligned}
Z_{A}^{(2)} & =\operatorname{Tr}\left[\rho_{A}^{\otimes 2}\right]=\operatorname{Tr}\left[\rho^{\otimes 2} \mathbb{F}^{(A)}\right] \\
& =\rho_{\bar{A}_{1} A_{1} \bar{B}_{1} B_{1}} \rho_{\bar{A}_{2} A_{2} \bar{B}_{2} B_{2}} \mathbb{F}_{\bar{A}_{1} A_{1} \bar{A}_{2} A_{2}}^{(A)} \mathbb{1}_{\bar{B}_{1} B_{1} \bar{B}_{2} B_{2}}^{(B)}, \\
Z_{0}^{(2)} & =\operatorname{Tr}\left[\rho^{\otimes 2}\right] \\
& =\rho_{\bar{A}_{1} A_{1} \bar{B}_{1} B_{1}} \rho_{\bar{A}_{2} A_{2} \bar{B}_{2} B_{2}} \mathbb{1}_{\bar{A}_{1} A_{1} \bar{A}_{2} A_{2}}^{(A)} \mathbb{1}_{\bar{B}_{1} B_{1} \bar{B}_{2} B_{2}}^{(B)} .
\end{aligned}
$$

\section{B. Self-averaging and large-bond-dimension limit}

Due to its random nature, our tensor network behaves analogously to systems with quenched disorder, characterized by self-averaging quantities, i.e., quantities for which the average over the random couplings produces typical configurations in the thermodynamic limit (see e.g., Ref. [19] and references therein).

In this sense, one can expect the entropy of the boundary state to be typical, namely to be arbitrarily well approximated by its expectation value, in the large-bond-dimension limit, namely for $D \gg 1$.

Following Ref. [12], we then consider the expansion of the expectation value $\mathbb{E}(\cdot)$ of the entropy average in powers of the fluctuations $\delta Z_{A}^{(2)}=Z_{A}^{(2)}-\mathbb{E}\left(Z_{A}^{(2)}\right)$ and $\delta Z_{0}^{(2)}=$ $Z_{0}^{(2)}-\mathbb{E}\left(Z_{0}^{(2)}\right)$, so that

$$
\begin{aligned}
\mathbb{E}\left(S_{N}(A)\right) & =-\mathbb{E}\left(\log \frac{\mathbb{E}\left(Z_{A}^{(2)}\right)+\delta Z_{A}^{(2)}}{\mathbb{E}\left(Z_{0}^{(2)}\right)+\delta Z_{0}}\right) \\
& =-\log \frac{\mathbb{E}\left(Z_{A}^{(2)}\right)}{\mathbb{E}\left(Z_{0}^{(2)}\right)}+\text { fluctuations. }
\end{aligned}
$$

For large enough bond dimensions $D$, building on the proof given in Ref. [12], as a direct consequence of the concentration of measure phenomenon [20], we assume in our case that the statistical fluctuations around the average value in Eq. (36) are exponentially suppressed. Within this assumption, we can approximate the second Rényi entropy with the logarithm of the ratio between the averages of $Z_{A}^{(2)}$ and $Z_{0}^{(2)}$,

$$
\mathbb{E}\left(S_{2}(A)\right) \simeq-\log \frac{\mathbb{E}\left(Z_{A}^{(2)}\right)}{\mathbb{E}\left(Z_{0}^{(2)}\right)} .
$$

We proceed then by computing $\mathbb{E}\left(Z_{A}^{(2)}\right)$ and $\mathbb{E}\left(Z_{0}^{(2)}\right)$ separately.

\section{Random state averaging}

Let us first consider the case without the gauge symmetry (10) for a given graph with only one node. The corresponding density matrix is

$$
\rho_{\mu \bar{\mu}} \equiv T_{\mu} \bar{T}_{\bar{\mu}} .
$$

Consider $N$ copies of the density matrix $\rho^{\otimes N}$. If $T$ is uniformly distributed, then the average $\rho^{\otimes N}$ over $T$ is given by

$$
\mathbb{E}_{T}\left(\rho^{\otimes N}\right) \equiv \int_{\mathrm{U}\left(D^{v}\right)} \mathrm{d} U \prod_{s=1}^{N}\left(U T^{0}\right)_{\mu^{(s)}}{\overline{\left(U T^{0}\right)_{\bar{\mu}^{(s)}}}}
$$

Because of Schur's lemma, since $\mathbb{}_{D^{v}}$ is the irrep of $\mathrm{U}\left(D^{v}\right)$, the result of the average is the identity matrix on the symmetric subspace of $\llbracket_{D^{v}}^{\otimes n}$. When $\operatorname{Tr} \rho=1$, the result is then

$$
\begin{aligned}
\mathbb{E}_{T}\left(\rho^{\otimes N}\right) & =\frac{1}{D^{v}[N]} \sum_{\pi \in \mathcal{S}_{N}} \prod_{s=1}^{N} \delta_{\mu^{(s)} \bar{\mu}^{(\pi(s))}} \\
& \equiv \frac{1}{D^{v}[N]} \sum_{\pi \in \mathcal{S}_{N}} \mathbb{P}\left(\pi ; N, D^{v}\right)
\end{aligned}
$$

where

$$
D^{v}[N] \equiv D^{v}\left(D^{v}+1\right) \ldots\left(D^{v}+N-1\right) .
$$

$\mathcal{S}_{N}$ is the symmetric group on $N$ objects and

$$
\mathbb{P}\left(\pi ; N, D^{v}\right) \equiv \prod_{s=1}^{N} \delta_{\mu^{(s)} \bar{\mu}^{(\pi(s))}}
$$


which is the representation matrix of $\pi \in \mathcal{S}_{N}$ on $\mathbb{H}_{D^{v}}^{\otimes N}$. Using the gauge (8) or (13), we can get the relation between representations on $\mathbb{H}_{D^{v}}$ and $\mathbb{U}_{D}^{\otimes v}$

$$
\delta_{\mu^{(s)} \bar{\mu}^{(\pi(s))}} \equiv \prod_{a=1}^{v} \delta_{\lambda_{a}^{(s)}\left(\bar{\lambda}_{a}^{((s))}\right.}
$$

Then we have

$$
\mathbb{P}\left(\pi ; N, D^{v}\right)=\prod_{a=1}^{v} \mathbb{P}\left(\pi_{a} ; N, D\right)
$$

If $T$ is a random vector, with Gaussian distribution, then the average is

$$
\mathbb{E}_{T}\left(\rho^{\otimes N}\right) \equiv \int \mathcal{D} T \mathrm{e}^{-\beta|T|^{2}} \prod_{s=1}^{N} T_{\mu^{(s)}} \bar{T}_{\bar{\mu}^{(s)}}
$$

If we ask that $\mathbb{E}_{T}(\rho)=\mathbb{1} / D^{v}$ and $T=x \hat{T}$, where $|\hat{T}|=1$, then $\beta=D^{v}$ and the average $\mathbb{E}_{T}\left(\rho^{\otimes N}\right)$ becomes

$$
\begin{aligned}
& \int \mathrm{d} x|x|^{2 N} \mathrm{e}^{-D^{v}|x|^{2}} \int \mathrm{d} U \prod_{s=1}^{N}\left(U \hat{T}^{0}\right)_{\mu^{(s)}} \overline{\left(U \hat{T}^{0}\right)_{\bar{\mu}^{(s)}}} \\
& =\left(D^{v}\right)^{-N} \sum_{\pi \in \mathcal{S}_{N}} \prod_{s=1}^{N} \delta_{\mu^{(s)} \bar{\mu}^{(\pi(s))}} .
\end{aligned}
$$

Now let us consider the case with the symmetry (10). The corresponding density matrix is

$$
\rho_{\mu \mu^{\prime}} \equiv T_{\mu} \bar{T}_{\bar{\mu}}=T_{\mu_{i}} \bar{T}_{\bar{\mu}_{\bar{i}}} \equiv \tilde{\rho}_{\mu_{i} \bar{\mu}_{\bar{i}}} .
$$

The expression for the $N$ copies of the density matrix reads

$$
\rho^{\otimes N}=\prod_{s=1}^{N} \tilde{\rho}_{\mu_{i(s)} \bar{\mu}_{\bar{i}(s)}}=\prod_{s=1}^{N} T_{\mu_{i(s)}} \bar{T}_{\bar{\mu}_{\bar{i}(s)}} .
$$

If $T$ is uniformly distributed, then the average of $\rho^{\otimes N}$ over $T$ is

$$
\mathbb{E}_{T}\left(\rho^{\otimes N}\right)=\int_{\mathrm{U}\left(D^{\nu-1}\right)} \mathrm{d} U \prod_{s=1}^{N}\left(U T^{0}\right)_{\mu_{i(s)}} \overline{\left(U T^{0}\right)_{\bar{\mu}_{\bar{i}(s)}}} .
$$

As shown in the first case, the result of the integral is the identity matrix in the symmetric subspace of $\mathbb{T}_{D^{v-1}}^{\otimes N}$

$$
\begin{aligned}
\mathbb{E}_{T}\left(\rho^{\otimes N}\right) & \propto \sum_{\pi \in \mathcal{S}_{N}} \prod_{s=1}^{N} \delta_{\mu_{i(s)} \bar{\mu}_{\bar{i}(\pi(s))}} \\
& \equiv \sum_{\pi \in \mathcal{S}_{N}} \mathbb{P}_{\{i(s)\}\{\bar{i}(s)\}}\left(\pi ; N, D^{v-1}\right)
\end{aligned}
$$

where $\mathbb{P}_{\{i(s)\}\{\bar{i}(s)\}}\left(\pi ; N, D^{v-1}\right)$ is the representation matrix of $\pi \in \mathcal{S}_{N}$ on $\mathbb{T}_{D^{v-1}}^{\otimes N}$ with a set of $\{i(s)\}\{\bar{i}(s)\}$. Similarly, when $T_{\mu}$ is a Gaussian vector, the result of the average is the same as the above equation up to a normalization. The details of the matrix in Eq. (50) are given in the Appendix.

By using the gauge (13), one can show the relation between the representations $\mu_{i}$ and $\lambda_{a}$. Because of Eq. (13), $\delta_{\mu_{i(s)} \bar{\mu}_{i\left(s^{\prime}\right)}}$ is not zero only when

$$
\left[\lambda_{a}(s)-i(s)\right]_{D}=\left[\bar{\lambda}_{a}\left(s^{\prime}\right)-\bar{i}\left(s^{\prime}\right)\right]_{D}
$$

Because of the modular rules (12), the above equation can be rewritten as

$$
\left[\lambda_{a}(s)-\bar{\lambda}_{a}\left(s^{\prime}\right)\right]_{D}=\left[i(s)-\bar{i}\left(s^{\prime}\right)\right]_{D}
$$

If $\left[i(s)-\bar{i}\left(s^{\prime}\right)\right]_{D}=\ell \in \mathbb{Z} / D$, then

$$
\delta_{\mu_{i(s)} \bar{H}_{\bar{i}\left(s^{\prime}\right)}}=\prod_{a=1}^{v} \delta_{\left[\lambda_{a}(s)-\bar{\lambda}_{a}\left(s^{\prime}\right)\right]_{D}, \ell}=\prod_{a=1}^{v} \delta_{\left[\lambda_{a}(s)-\ell\right]_{D}, \bar{\lambda}_{a}\left(s^{\prime}\right)} .
$$

Notice that $\ell$ is a uniform shift for all legs of each node as long as the step $\left[i(s)-\bar{i}\left(s^{\prime}\right)\right]_{D}=\ell$ is fixed. Finally, we define for later use the trace on a tensor $\mathbb{T}_{\left\{\mu^{(s)}\right\}\left\{\bar{\mu}^{(s)}\right\}}$, as

$$
\operatorname{Tr} \mathbb{T}=\mathbb{T}_{\left\{\mu^{(s)}\right\}\left\{\bar{\mu}^{(s)}\right\}} \prod_{s=1}^{N} \delta_{\mu^{(s)} \bar{\mu}^{(s)}}
$$

which becomes, with the symmetry (10),

$$
\operatorname{Tr} \mathbb{T}=\sum_{\substack{\{i(s)\} \\\langle i(s)\}}} \mathbb{T}_{\left\{\mu_{i(s)}\right\}\left\{\bar{\mu}_{\bar{i}(s)}\right\}} \prod_{s=1}^{N} \delta_{\mu_{i(s)} \bar{\mu}_{\bar{i}(s)}} \delta_{i(s) \bar{i}(s)} .
$$

\section{Second Renyi entropy with symmetry constraint}

Coming back to the case of $N=2$, we can now explicitly write down the expression for the average of the single symmetric tensor defined in Eq. (10),

$$
\mathbb{E}_{T}\left(\rho_{T_{n}}^{\otimes 2}\right)=\frac{1}{D^{2} D^{v-1}[2]} \sum_{\substack{m(1) \\ m(2)}}\left(\mathbb{1}_{m(1) m(2)}+\mathbb{F}_{m(1) m(2)}\right) .
$$

Given Eq. (41), $D^{v-1}[2]=D^{v-1}\left(D^{v-1}+1\right)$, we can define the normalization as $\mathbb{D}_{2}=D^{2} D^{v-1}[2]$. Therefore, for the density matrix of a tensor network with $N$ nodes we have 


$$
\begin{aligned}
\mathbb{E}_{T}\left(\rho^{\otimes 2}\right)= & \operatorname{Tr}_{C}\left[\underset{n}{\otimes} \mathbb{E}_{T}\left(\rho_{T_{n}}^{\otimes 2}\right) \rho_{M}^{\otimes 2}\right] \\
= & \frac{1}{\mathbb{D}_{2}^{N}} \operatorname{Tr}_{C}\left[\bigotimes _ { n } ^ { N } \sum _ { m _ { n } ( 1 ) m _ { n } ( 2 ) } \left(\mathbb{1}_{m_{n}(1) m_{n}(2)}\right.\right. \\
& \left.\left.+\mathbb{F}_{m_{n}(1) m_{n}(2)}\right) \rho_{M}^{\otimes 2}\right] \\
= & \frac{1}{\mathbb{D}_{2}^{N}} \sum_{\left\{m_{n}(1)\right\}\left\{m_{n}(2)\right\}} \\
& \times \operatorname{Tr}_{C}\left[{ }_{n}^{\otimes \mathbb{1}_{m_{n}}(1) m_{n}(2)}{\underset{n^{\prime}}{\otimes}}_{\left.\mathbb{F}_{n^{\prime}}(1) m_{n^{\prime}}(2) \rho_{M}^{\otimes 2}\right]}\right.
\end{aligned}
$$

In the case of a network of $N$ nodes, the above sum is naively given by a sum of $\left(2 D^{2}\right)^{N}$ terms, with $2 D^{2}$ choices for each node, but with several terms vanishing.

In order to calculate Eq. (56) explicitly, we consider the cases of internal and boundary links separately. For any internal links connecting two nodes, we find the following three main cases:

(1) $\mathbb{1}_{m(1) m(2)}$ and $\mathbb{1}_{m^{\prime}(1) m^{\prime}(2)}$,

$$
\operatorname{Tr}\left[\mathbb{1}_{m(1) m(2)} \rho_{M_{\ell}}^{\otimes 2} \mathbb{1}_{m^{\prime}(1) m^{\prime}(2)}\right]=D^{2} \delta_{m(1) m^{\prime}(1)} \delta_{m(2) m^{\prime}(2)} .
$$

(2) $\mathbb{F}_{m(1) m(2)}$ and $\mathbb{F}_{m^{\prime}(1) m^{\prime}(2)}$,

$$
\operatorname{Tr}\left[\mathbb{F}_{m(1) m(2)} \rho_{M_{\ell}}^{\otimes 2} \mathbb{F}_{m^{\prime}(1) m^{\prime}(2)}\right]=D^{2} \delta_{m(1) m^{\prime}(1)} \delta_{m(2) m^{\prime}(2)} .
$$

(3)

$$
\begin{aligned}
& \mathbb{1}_{m(1) m(2)} \text { and } \mathbb{F}_{m^{\prime}(1) m^{\prime}(2)}, \\
& \operatorname{Tr}\left[\mathbb{1}_{m(1) m(2)} \rho_{M_{\ell}}^{\otimes 2} \mathbb{F}_{m^{\prime}(1) m^{\prime}(2)}\right] \\
& \quad=D \delta_{[m(1)+m(2)]_{D},\left[m^{\prime}(1)+m^{\prime}(2)\right]_{D}}
\end{aligned}
$$

On the boundary of region $A$, for $\mathbb{F}^{(A)}=\mathbb{F}_{00}$ at one end of the boundary link, we have instead only two cases:

(1) $\mathbb{F}^{(A)}$ and $\mathbb{F}_{m^{\prime}(1) m^{\prime}(2)}$,

$$
\begin{aligned}
\operatorname{Tr}\left[\mathbb{F}^{(A)} \rho_{M_{\ell}}^{\otimes 2} \mathbb{F}_{m^{\prime}(1) m^{\prime}(2)}\right] & =\operatorname{Tr}\left[\mathbb{F}_{00} \rho_{M_{\ell}}^{\otimes 2} \mathbb{F}_{m^{\prime}(1) m^{\prime}(2)}\right] \\
& =D^{2} \delta_{0 m^{\prime}(1)} \delta_{0 m^{\prime}(2)} .
\end{aligned}
$$

(2) $\mathbb{F}^{(A)}$ and $\mathbb{1}_{m^{\prime}(1) m^{\prime}(2)}$,

$$
\begin{aligned}
\operatorname{Tr}\left[\mathbb{F}^{(A)} \rho_{M_{\ell}}^{\otimes 2} \mathbb{1}_{m^{\prime}(1) m^{\prime}(2)}\right] & =\operatorname{Tr}\left[\mathbb{F}_{00} \rho_{M_{\ell}}^{\otimes 2} \mathbb{1}_{m^{\prime}(1) m^{\prime}(2)}\right] \\
& =D \delta_{0,\left[m^{\prime}(1)+m^{\prime}(2)\right]_{D}}
\end{aligned}
$$

Finally, on the boundary of region $B$, where $\mathbb{1}^{(B)}=\mathbb{1}_{00}$ at one end of the boundary link, we find two cases:

(1) $\mathbb{1}^{(B)}$ and $\mathbb{F}_{m^{\prime}(1) m^{\prime}(2)}$,

$$
\begin{aligned}
\operatorname{Tr}\left[\mathbb{1}^{(B)} \rho_{M_{\ell}}^{\otimes 2} \mathbb{F}_{m^{\prime}(1) m^{\prime}(2)}\right] & =\operatorname{Tr}\left[\mathbb{1}_{00} \rho_{M_{\ell}}^{\otimes 2} \mathbb{F}_{m^{\prime}(1) m^{\prime}(2)}\right] \\
& =D \delta_{0,\left[m^{\prime}(1)+m^{\prime}(2)\right]_{D}}
\end{aligned}
$$

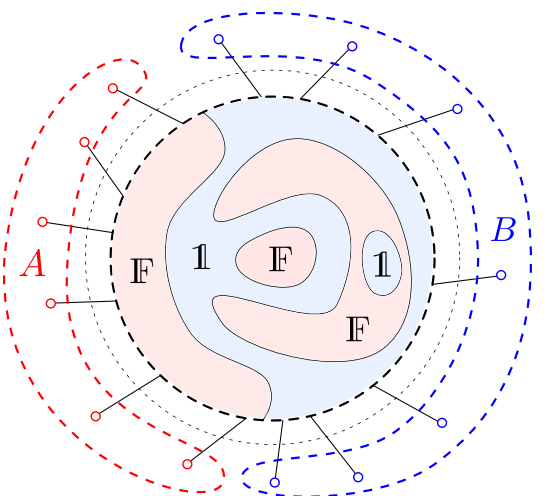

FIG. 2. The network with the boundary conditions $A=\mathbb{F}^{(A)}$ and $B=\mathbb{1}^{(B)}$ is divided into regions. The nodes in the red regions are associated with $\mathbb{F}$ and the ones in the blue regions are associated with 1 .

(2) $\mathbb{1}^{(B)}$ and $\mathbb{1}_{m^{\prime}(1) m^{\prime}(2)}$,

$$
\begin{aligned}
\operatorname{Tr}\left[\mathbb{1}^{(B)} \rho_{M_{\ell}}^{\otimes 2} \mathbb{1}_{m^{\prime}(1) m^{\prime}(2)}\right] & =\operatorname{Tr}\left[\mathbb{1}_{00} \rho_{M_{\ell}}^{\otimes 2} \mathbb{1}_{m^{\prime}(1) m^{\prime}(2)}\right] \\
& =D^{2} \delta_{0 m^{\prime}(1)} \delta_{0 m^{\prime}(2)} .
\end{aligned}
$$

Notice how the different scaling behavior seen in Eqs. (57)-(63) gives a good idea as to why connected patterns form. Connected configurations are characterized by a higher degree of divergence, and hence they are ultimately favored from an entropic point of view.

\section{E. Remarks on the calculation}

With the general scaling behavior in hand, the averaging $Z_{A}^{(2)}$ and $Z_{0}^{(2)}$ over $T$ is equivalent to defining a class of networks where to each node is assigned a matrix $\mathbb{1}_{m(1) m(2)}$ or a matrix $\mathbb{F}_{m(1) m(2)}$, while the two boundary regions are respectively labeled by matrices $\mathbb{F}^{(A)}$ and $\mathbb{1}^{(B)}$ for $Z_{A}^{(2)}$, and $\mathbb{1}^{(A)}$ and $\mathbb{1}^{(B)}$ for $Z_{0}^{(2)}$.

We find that, for all matrices $\mathbb{1}_{m(1) m(2)}$ and $\mathbb{F}_{m(1) m(2)}$, the ones with $[m(1)+m(2)]_{D} \neq 0$ will never contribute to $Z_{A}^{(2)}$ and $Z_{0}^{(2)}$. Indeed, if one node has $[m(1)+m(2)]_{D} \neq 0$, it will make its neighboring node satisfy $[m(1)+m(2)]_{D} \neq 0$, and these nodes will force their neighboring nodes to satisfy $[m(1)+m(2)]_{D} \neq 0$. Because all nodes connect to the boundary via a certain number of links, the consequence is that the boundary should be $[m(1)+m(2)]_{D} \neq 0$, but we have assumed that the boundary is assigned by $\mathbb{1}_{00}$ or $\mathbb{F}_{00}$, i.e., $[m(1)+m(2)]_{D}=0$. Therefore, none of the matrices at each node can satisfy $[m(1)+m(2)]_{D} \neq 0$. Therefore, the full calculation can be reduced to considering only matrices $\mathbb{F}_{m(1) m(2)}$ and $\mathbb{1}_{m(1) m(2)}$ with $[m(1)+m(2)]_{D}=0$.

If a node is $\mathbb{F}_{m(1) m(2)}$, then its neighboring nodes can only be $\mathbb{F}_{m^{\prime}(1) m^{\prime}(2)}$ or $\mathbb{1}_{m^{\prime}(1) m^{\prime}(2)}$ with $\left[m^{\prime}(1)+m^{\prime}(2)\right]_{D}=0$. So the network ends up being divided into several regions, 


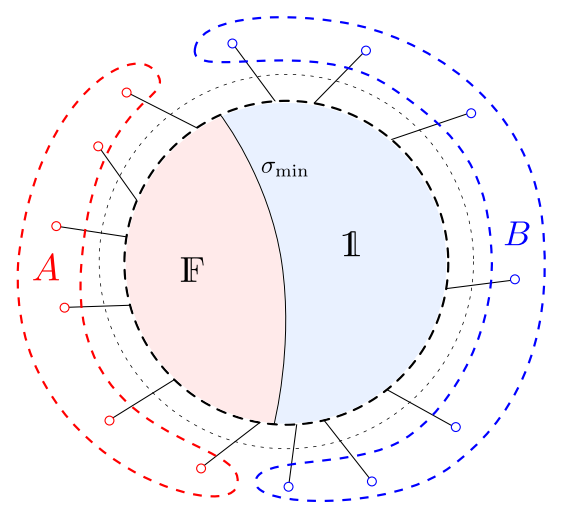

FIG. 3. The pattern with only the minimal surface $\sigma_{\min }$. Boundary condition: $A=\mathbb{F}^{(A)}$ and $B=\mathbb{1}^{(B)}$.

wherein all nodes are associated with the same matrix. If a region is associated with $\mathbb{F}_{m(1) m(2)}$, its neighboring region can only be associated with $\mathbb{1}_{m^{\prime}(1) m^{\prime}(2)}$, and vice versa. An example is shown in Fig. 2. In this sense, each region ends up being labeled with $\mathbb{F}$ or 1 and the boundaries between these regions can be interpreted as domain walls [12]. Each link crossing a domain wall is then assigned with $\mathbb{F}$ at one end and with 1 at the other.

As shown in Fig. 2, such domain walls form different global patterns $\mathcal{P}$ for the network. For a given pattern, changing a region's label from $\mathbb{F}_{m(1) m(2)}$ to $\mathbb{F}_{m^{\prime}(1) m^{\prime}(2)}$ will not change the value of its corresponding term in $Z_{A}^{(2)}$ or $Z_{0}^{(2)}$. This implies a degeneracy. The degeneracy of the region that does not connect to the boundary is $D$, which is the number of possible choices of the pair $(m(1), m(2))$. Therefore we have

$$
\begin{aligned}
& \mathbb{E}_{T}\left(Z_{A}^{(2)}\right)=\frac{1}{\mathbb{D}_{2}^{N}} \sum_{\mathcal{P}_{A}} d_{\mathcal{P}_{A}} Z_{\mathcal{P}_{A}}^{(2)}, \\
& \mathbb{E}_{T}\left(Z_{0}^{(2)}\right)=\frac{1}{\mathbb{D}_{2}^{N}} \sum_{\mathcal{P}_{0}} d_{\mathcal{P}_{0}} Z_{\mathcal{P}_{0}}^{(2)}
\end{aligned}
$$

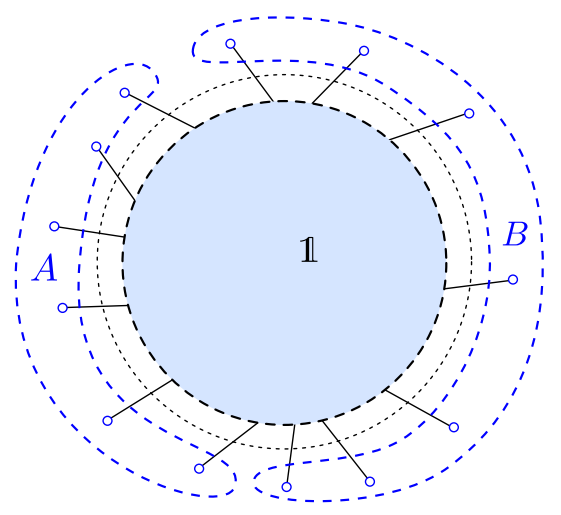

FIG. 4. The pattern without the minimal surface. Boundary condition: $A=\mathbb{1}^{(A)}$ and $B=\mathbb{1}^{(B)}$. where $d_{\mathcal{P}}$ is the degeneracy of the pattern, which is the product of the degeneracies of each region in this pattern. $Z_{\mathcal{P}}^{(2)}$ is given as

$$
Z_{\mathcal{P}}^{(2)}=D^{2\left(L-L_{\mathcal{P}}\right)} D^{L_{\mathcal{P}}}=D^{2 L-L_{\mathcal{P}}}
$$

where $L$ is the total link number in a given network $\mathcal{N}$, including links across $\partial \mathcal{N} ; L_{\mathcal{P}}$ is the number of links across the domain walls in $\mathcal{P}$.

\section{F. Holographic behavior and Ryu-Takayanagi formula}

The main contribution of $\mathbb{E}_{T}\left(Z_{A}^{(2)}\right)$ is given by the pattern with the least number of links through the domain walls. We call the domain wall with the least number of links the minimal surface. One can show that this is true even after the degeneracy $d_{\mathcal{P}}$ is taken into account.

In fact, all patterns can be generated from the minimal surface one by deforming the minimal surface or adding new regions. Starting from the pattern corresponding to a minimal surface, any deformation will lead to a surface which is not minimal. Adding a new region will multiply $D$ to the degeneracy $d_{\mathcal{P}}$, while will divide $D^{v}$ to $Z_{\mathcal{P}}$, where $v>1$ is the number of boundary links of the new region, and thus in total one has to consider the product $D^{1-v}<1$ to the original $Z_{\mathcal{P}}^{(2)}$. This gives a contribution that is smaller than the original one. So the main contribution to $\mathbb{E}_{T}\left(Z_{A}^{(2)}\right)$ necessarily comes from the minimal surface pattern. In this pattern there are only two regions, which are labeled by $\mathbb{F}^{(A)}$ and $\mathbb{1}^{(B)}$, respectively, as shown in Fig. $3^{3}$ :

$$
\mathbb{E}_{T}\left(Z_{A}^{(2)}\right)=\frac{1}{\mathbb{D}_{2}^{N}} D^{2 L-L_{\min }}\left(1+O\left(D^{-1}\right)\right) .
$$

In this sense, in agreement with the results in Ref. [12], we find that the main contribution of $\mathbb{E}_{T}\left(Z_{0}^{(2)}\right)$ is given by the pattern without any domain wall. This is because its boundary condition is $\mathbb{1}^{(A)}=\mathbb{1}^{(B)}=\mathbb{1}_{00}$. Such a pattern exists and all its nodes are assigned $\mathbb{1}_{00}$, as shown in Fig. 4 . Then

$$
\mathbb{E}_{T}\left(Z_{0}^{(2)}\right)=\frac{1}{\mathbb{D}_{2}^{N}} D^{2 L}\left(1+O\left(D^{-1}\right)\right) .
$$

Finally, collecting the above results, we find that the leading contribution to $S_{2}$ is given as

$$
\mathbb{E}_{T}\left(\mathrm{e}^{-S_{2}}\right) \sim \frac{\mathbb{E}_{T}\left(Z_{A}^{(2)}\right)}{\mathbb{E}_{T}\left(Z_{0}^{(2)}\right)}=D^{-L_{\min }}\left(1+O\left(D^{-1}\right)\right),
$$

which gives, when $D \gg 1$,

$$
S_{2}=L_{\min } \ln D+O\left(D^{-1}\right) .
$$

This is the Ryu-Takayanagi formula for $S_{2}$.

\footnotetext{
${ }^{3} \mathrm{~A}$ more extensive description of the configuration pattern can be found in Ref. [12] as well as in a related work by the authors [6].
} 
The $N$ th Rényi entropy $S_{N}$ can be calculated in a similar way, and with an analogous result.

\section{CONCLUSIONS}

We have computed the Rényi entropy and derived the Ryu-Takayanagi entropy formula, for random tensor networks with an additional gauge invariance property. This is an interesting extension of existing derivations. On the one hand it shows the generality of the holographic behavior of (random) tensor network states, and thus their role in the entanglement/geometry correspondence, and confirms their interest also for applications in a quantum gravity context. On the other hand, the type of gauge symmetry we imposed is suggested by the correspondence with GFT and LQG states, and it is indeed required for the exact matching with the spin network states used in these quantum gravity approaches. Thus our results will facilitate the application of techniques from quantum gravity to quantum manybody systems (beyond the AdS/CFT framework), and the exploration of the same entanglement/geometry correspondence in new quantum gravity contexts.

\section{ACKNOWLEDGMENTS}

M. Z. acknowledges support from the A. von Humboldt Foundation.

\section{APPENDIX: STRUCTURE OF THE MATRIX OF $\mathbb{E}_{T}\left(\rho^{\otimes n}\right)$}

In this appendix we analyze the structure of the matrix in Eq. (50)

$$
\mathbb{M} \equiv \sum_{\pi \in \mathcal{S}_{n}} \prod_{s=1}^{n} \delta_{\mu_{i(s)} \bar{\mu}_{\bar{i}(\pi(s))}}=\sum_{\pi \in \mathcal{S}_{n}} \mathbb{P}_{\{i(s)\}\{\bar{i}(s)\}}\left(\pi ; n, D^{v-1}\right)
$$

The sum over $\mathbb{P}\left(\pi ; n, D^{v-1}\right)$ is proportional to the projector operator $\mathbb{P}_{\mathrm{sym}}^{n, D^{v-1}}$ which projects vectors in $\mathbb{U}_{D^{v-1}}^{\otimes n}$ into its symmetric subspace,

$$
\mathbb{P}_{\mathrm{sym}}^{n, D^{v-1}}=\frac{1}{n !} \sum_{\pi \in \mathcal{S}_{n}} \mathbb{P}\left(\pi ; n, D^{v-1}\right)
$$

Given a set of $\{i(s)\}=\{i(1), i(2), \ldots, i(n)\}$ and $\{\bar{i}(s)\}=$ $\{\bar{i}(1), \bar{i}(2), \ldots, \bar{i}(n)\}$ where $i(s)$ and $\bar{i}(s)$ are from 0 to $D-1$, there is a projector $\mathbb{P}_{\mathrm{sym}}^{n, D^{v-1}}$, which is a $D^{(v-1) n} \times$ $D^{(v-1) n}$ matrix. Write Eq. (50) as a matrix, with $\{i(s)\}$ labeling its rows and $\{\bar{i}(s)\}$ labeling its columns:

$$
\mathbb{M}=n !\left(\begin{array}{ccc}
\mathbb{P}_{\mathrm{sym}}^{n, D^{v-1}} & \cdots & \mathbb{P}_{\mathrm{sym}}^{n, D^{v-1}} \\
\vdots & \ddots & \vdots \\
\mathbb{P}_{\mathrm{sym}}^{n, D^{v-1}} & \cdots & \mathbb{P}_{\mathrm{sym}}^{n, D^{v-1}}
\end{array}\right)
$$

This matrix is a $D^{n} \times D^{n}$ block matrix.

The trace of $\mathbb{M}$ is

$$
\begin{aligned}
\operatorname{TrM} & =\sum_{\{i(s)\}} \sum_{\pi \in \mathcal{S}_{n}} \prod_{s=1}^{n} \delta_{\mu_{i(s)} \bar{\mu}_{\bar{i}(\pi(s))}} \delta_{\mu_{i(s)} \bar{\mu}_{\bar{i}(s)}} \delta_{i(s) \bar{i}(s)} \\
& =\sum_{\{i(s)\}} \sum_{\pi \in \mathcal{S}_{n}} \prod_{s=1}^{n} \delta_{\mu_{i(s)} \bar{\mu}_{i(\pi(s))}} \delta_{\mu_{i(s)} \bar{\mu}_{i(s)}} \\
& =\sum_{\{i(s)\}} \sum_{\pi \in \mathcal{S}_{n}} \prod_{s=1}^{n} \delta_{\mu_{i(s)} \mu_{i(\pi(s))}} \\
& =\sum_{\{i(s)\}} n ! \operatorname{Tr} \mathbb{P}_{\mathrm{sym}}^{n, D^{v-1}} \\
& =D^{n} D^{v-1}[n]
\end{aligned}
$$

where we use the trace of the projector $\mathbb{P}_{\text {sym }}^{n}$

$$
\operatorname{Tr} \mathbb{P}_{\mathrm{sym}}^{n, D^{v-1}}=\left(\begin{array}{c}
D^{v-1}+n- \\
n
\end{array}\right)
$$

The matrix $\mathbb{M}$ can be written as a sum of matrices

$$
\begin{aligned}
\mathbb{M} & =\sum_{\pi \in \mathcal{S}_{n}} \prod_{s=1}^{n} \delta_{\mu_{i(s)} \bar{\mu}_{\bar{i}(\pi(s))}} \\
& =\sum_{\{m(s)\}} \sum_{\pi \in \mathcal{S}_{n}} \prod_{s=1}^{n} \delta_{\mu_{\left[i(s)-m(s) D_{D}\right.} \bar{\mu}_{\bar{i}(\pi(s))}} \delta_{i(s) \bar{i}(\pi(s))} \\
& =\sum_{\{m(s)\}} \sum_{\pi \in \mathcal{S}_{n}} \prod_{s=1}^{n} \delta_{\left[\mu^{(s)}-m(s) D^{v-1}\right]_{D^{v}}, \bar{\mu}^{(\pi(s))}} \\
& =\sum_{\{m(s)\}} \sum_{\pi \in \mathcal{S}_{n}} \prod_{s=1}^{n} \prod_{a=1}^{v} \delta_{\left[\lambda_{a}(s)-m(s)\right]_{D}, \bar{\lambda}_{a}(\pi(s))}
\end{aligned}
$$

Let us define a class of new matrices $\mathbb{P}(\pi)_{\{m(s)\}}$ as

$$
\begin{aligned}
\mathbb{P}(\pi)_{\{m(s)\}} & \equiv \prod_{s=1}^{n} \prod_{a=1}^{v} \delta_{\left[\lambda_{a}(s)-m(s)\right]_{D}, \bar{\lambda}_{a}(\pi(s))} \\
& =\prod_{s=1}^{n} \delta_{\left[\mu^{(s)}-m(s) D^{v-1}\right]_{D^{v}}, \bar{\mu}(\pi(s))} .
\end{aligned}
$$

Then the matrix $\mathbb{M}$ becomes

$$
\mathbb{M}=\sum_{\{m(s)\}} \sum_{\pi \in \mathcal{S}_{n}} \mathbb{P}(\pi)_{\{m(s)\}} .
$$

When $m(s)=0$ for all $s, \mathbb{P}(\pi)_{\{0\}}=\mathbb{P}\left(\pi ; n, D^{v}\right)$, the representation matrix of $\pi \in \mathcal{S}_{n}$ on $\mathbb{U}_{D^{v}}^{\otimes n}$. 
[1] R. Orus, Ann. Phys. (Amsterdam) 349, 117 (2014); J. C. Bridgeman and C. T. Chubb, J. Phys. A 50, 223001 (2017).

[2] J. I. Cirac and F. Verstraete, J. Phys. A 42, 504004 (2009); F. Verstraete, V. Murg, and J. I. Cirac, Adv. Phys. 57, 143 (2008); F. Pastawski, B. Yoshida, D. Harlow, and J. Preskill, J. High Energy Phys. 06 (2015) 149; G. Vidal, Phys. Rev. Lett. 101, 110501 (2008); S. Singh, R. N. C. Pfeifer, and G. Vidal, Phys. Rev. A 82, 050301 (2010); G. Evenbly and G. Vidal, J. Stat. Phys. 145, 891 (2011).

[3] D. Oriti, in Proceedings, Foundations of Space and Time: Reflections on Quantum Gravity, Cape Town, South Africa, 2009, edited by J. Murugan, A. Weltman, and G. F. R. Ellis (Cambridge University Press, Cambridge, England, 2012), p. 257; D. Oriti, arXiv:1408.7112; A. Baratin and D. Oriti, J. Phys. Conf. Ser. 360, 012002 (2012).

[4] R. Gurau and J. P. Ryan, SIGMA 8, 020 (2012); R. Gurau, SIGMA 12, 094 (2016); V. Rivasseau, SIGMA 12, 069 (2016).

[5] A. Ashtekar and J. Lewandowski, Classical Quantum Gravity 21, R53 (2004); C. Rovelli, Quantum Gravity (Cambridge University Press, Cambridge, England, 2004); T. Thiemann, Modern Canonical Quantum General Relativity (Cambridge University Press, Cambridge, England, 2007); A. Perez, Living Rev. Relativity 16, 3 (2013).

[6] G. Chirco, D. Oriti, and M. Zhang, arXiv:1701.01383.

[7] B. Dittrich, S. Mizera, and S. Steinhaus, New J. Phys. 18, 053009 (2016); C. Delcamp and B. Dittrich, Classical Quantum Gravity 34, 225006 (2017); B. Dittrich, F. C. Eckert, and M. Martin-Benito, New J. Phys. 14, 035008 (2012); B. Dittrich, E. Schnetter, C. J. Seth, and S. Steinhaus, Phys. Rev. D 94, 124050 (2016).

[8] A. Baratin and D. Oriti, Phys. Rev. Lett. 105, 221302 (2010); Phys. Rev. D 85, 044003 (2012); M. Han and T. Krajewski, Classical Quantum Gravity 31, 015009 (2014),
arXiv:1304.5626; M. Han and M. Zhang, Classical Quantum Gravity 30, 165012 (2013); M. Han and L.-Y. Hung, Phys. Rev. D 95, 024011 (2017); J. High Energy Phys. 11 (2017) 148.

[9] D. Oriti, Stud. Hist. Phil. Sci. B 46, 186 (2014); C. Cao, S. M. Carroll, and S. Michalakis, Phys. Rev. D 95, 024031 (2017); N. Seiberg, in The Quantum Structure of Space and Time: Proceedings of the 23rd Solvay Conference on Physics. Brussels, Belgium, 2005, edited by D. Gross, M. Henneaux, and A. Sevrin (World Scientific, Singapore, 2006), p. 163; D. Oriti, Proc. Sci., QG-PH2007 (2007) 030, arXiv:0710.3276; V. Rivasseau, AIP Conf. Proc. 1444, 18 (2011); D. Oriti, arXiv:1710.02807.

[10] S. Ryu and T. Takayanagi, Phys. Rev. Lett. 96, 181602 (2006).

[11] B. Swingle, Phys. Rev. D 86, 065007 (2012).

[12] P. Hayden, S. Nezami, X.-L. Qi, N. Thomas, M. Walter, and Z. Yang, J. High Energy Phys. 11 (2016) 009.

[13] D. N. Page, Phys. Rev. Lett. 71, 1291 (1993).

[14] J. M. Deutsch, Phys. Rev. A 43, 2046 (1991).

[15] M. Srednicki, Phys. Rev. E 50, 888 (1994).

[16] S. Singh, R. N. C Pfeifer, and G. Vidal, Phys. Rev. B 83, 115125 (2011); Y. Li, M. Han, D. Ruan, and B. Zeng, J. Phys. A 51, 175303 (2018).

[17] M. Nozaki, S. Ryu, and T. Takayanagi, J. High Energy Phys. 10 (2012) 193.

[18] E. R. Livine and D. R. Terno, Nucl. Phys. B741, 131 (2006); W. Donnelly, Phys. Rev. D 77, 104006 (2008); D. Oriti, Classical Quantum Gravity 33, 085005 (2016); G. Chirco, F. Mele, D. Oriti, and P. Vitale, Phys. Rev. D 97, 046015 (2018); L. Freidel, A. Perez, and D. Pranzetti, Phys. Rev. D 95, 106002 (2017).

[19] G. Parisi, Lett. Math. Phys. 88, 255 (2009).

[20] P. Hayden, D. Leung, and A. Winter, Commun. Math. Phys. 265, 95 (2006). 